\title{
Exploiting Randomness in Quantum Information Processing
}

\author{
Chiu Fan Lee* and Neil F. Johnson ${ }^{\dagger}$ \\ Center for Quantum Computation and Physics Department \\ Clarendon Laboratory, Oxford University \\ Parks Road, Oxford OX1 3PU, U.K.
}

October 25, 2018

\begin{abstract}
We consider how randomness can be made to play a useful role in quantum information processing - in particular, for decoherence control and the implementation of quantum algorithms. For a twolevel system in which the decoherence channel is non-dissipative, we show that decoherence suppression is possible if memory is present in the channel. Random switching between two potentially harmful noise sources can then provide a source of stochastic control. Such random switching can also be used in an advantageous way for the implementation of quantum algorithms.
\end{abstract}

${ }^{*}$ c.lee1@physics.ox.ac.uk

†n.johnson@physics.ox.ac.uk 


\section{Introduction}

Randomness and noise are typically seen as having a detrimental effect on the coherent evolution of a quantum system, and hence on the ability of the system to process quantum information [1]. For classical systems, the advent of phenomena such as stochastic resonance, Brownian ratchets and the Parrondo effect, have shown that noise may indeed play a helping role after all [2]. This opens up the intriguing question of whether randomness can play a useful role in quantum systems, in particular given the widespread current interest in quantum information schemes such as quantum computation [1].

Here we investigate how the intrinsic randomness of an open quantum system might actually be used to our advantage for quantum information processing. First we consider the role of randomness in suppressing and controlling decoherence (Secs. 2 and 3). For a twolevel system in which the decoherence channel is non-dissipative, we show that suppresion of decoherence is possible if memory is present in the channel. Random switching between two potentially harmful noise sources, can then provide a source of stochastic control. Second, we show how random switching can be used in an advantageous way for the implementation of quantum algorithms (Sec. 4).

\section{Stochastic decoherence}

Decoherence is a unique quantum phenomenon which results in a decay of the off-diagonal elements in a density matrix. Simply put, it is the following process:

$$
\rho_{0}:=\left(\begin{array}{cc}
a & b \\
b^{*} & c
\end{array}\right) \stackrel{t}{\mapsto}\left(\begin{array}{cc}
a & b^{\prime} \\
b^{\prime *} & c
\end{array}\right)
$$

where $\left|b^{\prime}\right|<|b|$. This results in the decay of superpositions of states into a probablistic mixture.

\section{$2.1 \quad$ Non-dissipative system}

We now study decoherence in a two-level system under the physical assumptions that the channel is (i) non-dissipative, and (ii) isolated, i.e. no entanglement is allowed between the system and the environment. This decoherence can be thought of as arising from the observers' limited knowledge of the channel, e.g. due to uncontrollable classical fluctuations. If we further assume discrete time evolution for simplic-

ity, the above assumptions imply that the final density matrix can be 
written as $\rho_{n}=U_{n} \cdots U_{1} \rho_{0} U_{1}^{\dagger} \cdots U_{n}^{\dagger}$ where

$$
U_{k}=\left(\begin{array}{cc}
e^{-i \theta_{k} / 2} & 0 \\
0 & e^{i \theta_{k} / 2}
\end{array}\right) .
$$

Decoherence can only occur if our knowledge of $\theta_{k}$ 's is uncertain. To prove that such a lack of knowledge will almost surely lead to decoherence, let us assume that the phase kicks $\theta$ are independent and identically distributed with probability distribution $P(\theta)$. We will then have

$$
\rho_{n}=\left(\begin{array}{cc}
a & b \gamma^{n} e^{-i n \phi} \\
b^{*} \gamma^{n} e^{i n \phi} & c
\end{array}\right)
$$

where

$$
\gamma e^{ \pm i \phi}:=\int_{\theta_{l o}}^{\theta_{h i}} e^{ \pm i \theta} P(\theta) d \theta
$$

Hence, $|\gamma| \leq 1$ and the equality is satisfied if and only if $P(\theta)=$ $\sum_{k} p_{k} \delta(\theta-a-k)$ for some constant $a$ [3]. This condition will only be met in exceptional circumstances - therefore stochastic decoherence will essentially always arise in such a system.

We now let $\tau_{0}$ be the interaction time and set $n=t / \tau_{0}$. Letting $P(\theta)$ be $\frac{\exp \left(\theta / \omega \tau_{1}\right)}{\omega \tau_{1}}$ for $\theta \geq 0$, we find $\gamma=\sqrt{1+\omega^{2} \tau_{1}^{2}}$ and $\phi=$ $\arctan \left(\omega \tau_{1}\right)$. This coincides with the main result in Ref. [ब] where the constant $\tau_{1}$ corresponds to the "time width of each event" 淉. On the other hand, by setting $P(\theta):=\frac{1}{\sigma \sqrt{2 \pi}} \exp \left[-\frac{(\theta-\mu)^{2}}{2 \sigma^{2}}\right]$ where $\mu:=\sin (\omega / \lambda)$ and $\sigma^{2}:=2(1-\cos (\omega / \lambda))$, we recover the result in Ref. [6] if we identify $\lambda$ with the "fundamental time of the universe" [6]. This observation makes sense because our assumptions are the most general ones. Indeed for any decay factor of the form $\left(\gamma e^{i \phi}\right)^{t / \tau_{0}}$ with $\gamma<1$, we could pick $P(\theta):=\frac{1}{\sigma \sqrt{2 \pi}} \exp \left[-\frac{(\theta-\mu)^{2}}{2 \sigma^{2}}\right]$ with $\mu:=\phi$ and $\sigma^{2}:=-2 \ln \gamma$.

Given that stochastic decoherence will almost always be present in a real-world quantum system, in accordance with the discussion above, one can ask whether a method can be devised to control it. Unfortunately, we can show that there is actually no way to suppress the stochastic decoherence discussed above if the channel has no memory. The justification of this statement is as follows. Let us assume that such an operation is feasible and let us call it $\mathcal{F}$, where

$$
\mathcal{F}:\left(\begin{array}{cc}
a & b \\
b^{*} & c
\end{array}\right) \mapsto\left(\begin{array}{cc}
a & b^{\prime} \\
b^{\prime *} & c
\end{array}\right)
$$

and where $\left|b^{\prime}\right|>|b|$ for a non-empty set of $b$ values. We require that $\mathcal{F}$ be non-dissipative, i.e. we don't allow dissipation in exchange for 
decoherence suppression. Hence

$$
\mathcal{F}\left(\left(\begin{array}{ll}
1 & 0 \\
0 & 0
\end{array}\right)\right)=\left(\begin{array}{ll}
1 & 0 \\
0 & 0
\end{array}\right) \quad ; \quad \mathcal{F}\left(\left(\begin{array}{ll}
0 & 0 \\
0 & 1
\end{array}\right)\right)=\left(\begin{array}{ll}
0 & 0 \\
0 & 1
\end{array}\right) .
$$

Letting

$$
\mathcal{F}\left(\left(\begin{array}{ll}
0 & 1 \\
0 & 0
\end{array}\right)\right)=\left(\begin{array}{ll}
\alpha & \beta \\
\gamma & \delta
\end{array}\right) \quad ; \mathcal{F}\left(\left(\begin{array}{ll}
0 & 0 \\
1 & 0
\end{array}\right)\right)=\left(\begin{array}{cc}
\alpha^{\prime} & \beta^{\prime} \\
\gamma^{\prime} & \delta^{\prime}
\end{array}\right),
$$

we see that for some $b,\left|\beta+e^{i \theta} \beta^{\prime}\right|>1$ where $e^{i \theta}=b^{*} / b$. But if we now take $\rho_{0}:=\frac{1}{\sqrt{2}}\left(\begin{array}{cc}1 & e^{i \theta / 2} \\ e^{-i \theta / 2} & 1\end{array}\right)$, then

$$
\mathcal{F}\left(\rho_{0}\right)=\frac{1}{\sqrt{2}}\left(\begin{array}{cc}
1 & \beta+e^{i \theta} \beta^{\prime} \\
\left(\beta+e^{i \theta} \beta^{\prime}\right)^{*} & 1
\end{array}\right)
$$

This latter quantity $\mathcal{F}\left(\rho_{0}\right)$ will not have positive eigenvalues because the off-diagonal elements have norms greater than 1 , thereby contradicting the fact that $\mathcal{F}$ is a superoperator. In other words, the above demonstration shows that there is no 'coherence booster' - even for particular states.

\subsection{Dissipative system}

Through the following example, we illustrate how a dissipative system may be modelled using a similar stochastic process. Consider the following channel:

$$
\mathcal{E}(\rho)=p E_{0} \rho E_{0}^{\dagger}+p E_{1} \rho E_{1}^{\dagger}+(1-p) R_{z} \rho R_{z}^{\dagger}
$$

where

$E_{0}:=\left(\begin{array}{cc}1 & 0 \\ 0 & \sqrt{1-\alpha}\end{array}\right), E_{1}:=\left(\begin{array}{cc}0 & \sqrt{\alpha} \\ 0 & 0\end{array}\right), R_{z}:=\left(\begin{array}{cc}e^{-i \theta / 2} & 0 \\ 0 & e^{i \theta / 2}\end{array}\right)$

Hence

$$
\mathcal{E}(\rho)=\left(\begin{array}{cc}
1-(1-\alpha p)(1-a) & b\left[p \sqrt{1-\alpha}+(1-p) e^{-i \theta}\right] \\
b^{*}\left[p \sqrt{1-\alpha}+(1-p) e^{i \theta}\right] & c(1-\alpha p)
\end{array}\right) .
$$

Keeping $p$ fixed and assuming $\alpha, \theta$ to be Gaussian and independent, gives

$$
\iint \mathcal{E}(\rho) P_{a d}(\alpha) P_{p d}(\theta) d \alpha d \theta=
$$




$$
\left(\begin{array}{cc}
1-\left(1-p \sqrt{\frac{4 \lambda_{a d}}{\pi}}\right)\left(1-|a|^{2}\right) & a b^{*}\left[p\left(1-\sqrt{\frac{\lambda_{a d}}{\pi}}\right)+(1-p) e^{-\lambda_{p d}}\right] \\
a^{*} b\left[p\left(1-\sqrt{\frac{\lambda_{a d}}{\pi}}\right)+(1-p) e^{\left.-\lambda_{p d}\right]}\right. & |b|^{2}\left(1-p \sqrt{\frac{4 \lambda_{a d}}{\pi}}\right)
\end{array}\right)
$$

where we have assumed $\lambda_{a d} \ll 1$, and a Taylor expansion has been used on the diagonal terms before integrating. In particular, if it is known that the relevant timescales satisfy $T_{1} \geq T_{2} / 2$ (see e.g. Ref. [5]), then we need

$$
p \leq \frac{1-e^{-\lambda_{p d}}}{1-e^{-\lambda_{p d}}+\sqrt{\lambda_{a d} / \pi}} .
$$

\section{Control of decoherence via random- ness}

Decoherence control is crucial to the success of quantum computation. We now study the possibility of controlling stochastic decoherence using further randomness.

\subsection{A vector-rotating game}

We start by introducing a classical vector-rotating game which shows a Parrondo-like effect [2]. This particular game motivates much of the later development of decoherence control and the discussion of algorithms.

Game A: Consider a wheel with a vector drawn from the center to the circumference, i.e. the vector is a radial line. Suppose the vector is originally vertical (i.e. $\theta=0$ ) and the player plays by calling a robot (A) to rotate the wheel. The robot can only rotate the wheel by 0 , $2 \pi / 3$ or $4 \pi / 3$ radians, with equal probabilities. The player wins if the vector ends up in the upper-half of the circle (i.e. $-\pi / 2 \leq \theta \leq \pi / 2$ ) and he loses otherwise. The game is continued by rotating the wheel from the previous position, i.e. without restoring the vector to the vertical position. The stationary states are such that the vector will end up at $\theta=0,2 \pi / 3$ or $4 \pi / 3$ with equal probabilities. Therefore this game is losing for the player and the rate of losing is $1 / 3$. In Parrondo's original game, the losing rate is smaller (i.e. $-2 \epsilon$ where $\epsilon \ll 1)$.

Game B: This is the same as game A, except that the robot (B) can now only rotate the wheel by $0,2 \pi / 7,4 \pi / 7,6 \pi / 7,8 \pi / 7,10 \pi / 7$, $12 \pi / 7$, with equal probabilities. Similar analysis as that for game A shows the player's losing rate is $1 / 7$. In Parrondo's original game, the losing rate is again smaller (i.e. $-11 \epsilon / 5$ where $\epsilon \ll 1$ ). 
Game $A \oplus B$ : The player now plays a combined game in which he randomly selects either $\mathrm{A}$ or $\mathrm{B}$ at each timestep. Operationally, one of the robots A or B is selected at random to rotate the wheel at each timestep. Simple geometric analysis shows that the vector can now end up in $3 \times 7=21$ different orientations, 11 of which are winning. The corresponding $21 \times 21$ transition matrix is doubly-stochastic and so the stationary distribution will be equally distributed among these 21 positions. Therefore the player now wins with probability $11 / 21 \approx$ $0.5238>1 / 2$. In Parrondo's original game, the winning rate was $1 / 80-21 \epsilon / 10$ as compared to the present, larger rate of $1 / 21$. It turns out there is nothing special about the numbers 3 and 7 chosen for this implementation. The games $\mathrm{A}$ and $\mathrm{B}$ are originally losing simply because $3=7=3 \bmod 4$, and the combined game becomes winning because $3 \times 7=1 \bmod 4$. Therefore, the above vector-rotating implementation of Parrondo's effect works equally well for all $m, n$ such that $(m, n)=1$ and $m=n=3 \bmod 4$. By the same method, we can therefore construct two losing games with rates $-1 / m<0$ and $-1 / n<0$ such that when they are combined at random, we obtain a winning game with rate $1 / m n>0$. One could also extend the Parrondo scheme to include random combinations of any even number of games.

\subsection{Stochastic control}

We showed earlier that it is impossible to control decoherence if the noise does not have any "memory". This then leads us to consider correlated phase kicks. Depending on the particular model employed, some of the control methods devised elsewhere might also work [7, 81. However, we choose here to focus on a stochastic suppression of decoherence which mimics some form of Parrondo effect [2].

Motivated by the classical vector-rotation game we introduced earlier, we consider two probability distributions $P_{A}, P_{B}$ which are correlated to the previous rotated angle $\left(\theta_{1}\right)$ in the following manner:

$$
\begin{aligned}
& P_{A}\left(\theta_{2} \mid \theta_{1}\right)= \begin{cases}\frac{1}{3}[\delta(0)+\delta(-\pi / 2)+\delta(\pi / 2)] & , \quad \theta_{1} \in\{-\pi / 2,0, \pi / 2\} \\
\delta(0) & , \quad \text { otherwise }\end{cases} \\
& P_{B}\left(\theta_{2} \mid \theta_{1}\right)= \begin{cases}\frac{1}{3}[\delta(\epsilon)+\delta(-3 \pi / 4)+\delta(\pi / 4)] & , \quad \theta_{1} \in\{-3 \pi / 4, \epsilon, \pi / 4\} \\
\delta(\epsilon) & , \quad \text { otherwise. }\end{cases}
\end{aligned}
$$

If $P_{A}$ is the only noise in the system and if we assume the initial angle of rotation is 0 , we will have

$$
P_{A}\left(\theta_{n}, \ldots, \theta_{1}\right)=\prod_{i} P_{A}\left(\theta_{i}\right)
$$


as the $\theta_{i}$ 's always lie in the set $\{-\pi / 2,0, \pi / 2\}$. Therefore,

$$
\gamma_{A} e^{ \pm i \phi_{A}}:=\int e^{ \pm i \theta} P_{A}(\theta) d \theta=\frac{1}{3}
$$

Similarly,

$$
\gamma_{B} e^{ \pm i \phi_{B}}:=\int e^{ \pm i \theta} P_{B}(\theta) d \theta=\frac{1}{3} e^{i \epsilon}
$$

with $\gamma_{A}=\gamma_{B}=1 / 3$.

Combining the two probability distributions at random gives

$$
P\left(\theta_{2} \mid \theta_{1}\right)= \begin{cases}\frac{1}{2} \delta(\epsilon)+\frac{1}{6}[\delta(0)+\delta(-\pi / 2)+\delta(\pi / 2)] & , \theta_{1} \in\{-\pi / 2,0, \pi / 2\} \\ \frac{1}{2} \delta(0)+\frac{1}{6}[\delta(\epsilon)+\delta(-3 \pi / 4)+\delta(\pi / 4)] & , \text { otherwise. }\end{cases}
$$

Since $R_{z}(\theta) R_{z}(\phi)=R_{z}(\phi) R_{z}(\theta)$, we can write $\rho_{n}$ as

$$
\int R_{z}\left(\theta_{1}\right) \cdots \int R_{z}\left(\theta_{n}\right) \rho_{0} R_{z}^{\dagger}\left(\theta_{n}\right) P\left(\theta_{n} \mid \theta_{n-1}\right) d \theta_{n} \cdots R_{z}^{\dagger}\left(\theta_{1}\right) P\left(\theta_{1}\right) d \theta_{1}
$$

We now define the following functions recursively:

$$
\begin{aligned}
f_{1}(\theta) & :=\int e^{i \phi} P(\phi \mid \theta) d \phi \\
f_{k+1}(\theta) & :=\int e^{i \phi} f_{k}(\phi) P(\phi \mid \theta) d \phi
\end{aligned}
$$

for $1 \leq k \leq n$. Here $\rho=f_{n}(0)$, assuming that the initial angle is 0 .

For the combined probability distribution $P$ above, we see that the angles of rotation can only take on six values, $\{-3 \pi / 4,-\pi / 2,0, \epsilon, \pi / 4, \pi / 2\}$. Furthermore, we can calculate the $f_{k}$ 's to be the following:

$$
\begin{gathered}
f_{1}: \begin{cases}\{-\pi / 2,0, \pi / 2\} & \mapsto e^{i \epsilon} / 2+1 / 6 \\
\{-3 \pi / 4, \epsilon, \pi / 4\} & \mapsto 1 / 2+e^{i \epsilon} / 6\end{cases} \\
f_{k+1}: \begin{cases}\{-\pi / 2,0, \pi / 2\} & \mapsto \frac{1}{2} f_{k}(0)+\frac{1}{6} f_{k}(\epsilon) \\
\{-3 \pi / 4, \epsilon, \pi / 4\} & \mapsto \frac{1}{2} f_{k}(\epsilon)+\frac{1}{6} f_{k}(0) .\end{cases}
\end{gathered}
$$

Letting $\epsilon$ go to zero and writing $e^{i \epsilon}$ as $1+\mathcal{O}(\epsilon)$, we see that the $f_{k}$ 's always output $2 / 3+\mathcal{O}(\epsilon)$. An immediate consequence is that $\rho_{n}^{(10)}$ has an exponential decay factor of $2 / 3+\mathcal{O}(\epsilon)$. This is an improvement over the value $1 / 3$, which is the decay factor if we were to consider noise $P_{A}$ and noise $P_{B}$ separately. This result is reminiscent of the Parrondo effect discussed earlier for classical systems [2]. We note in passing that Mancini et al [9] have also devised a stochastic scheme to control quantum coherence. Although these authors invoke a memoryless modulation of the cavity length, their model is dissipative. More specifically, their only source of decoherence is the loss of photons hence their results are fundamentally different from the present case of a non-dissipative channel. 


\section{A stochastic algorithm}

We now turn to an example involving quantum algorithms, in which cooperating with randomness may be a better strategy than trying to fight it. We consider a game where the player's goal is to obtain (i.e. measure with a high probability) a fixed, unknown number $\alpha$ in as few timesteps as possible. Here $0 \leq \alpha \leq 2^{n}-1$. The initial state has the form $|\psi\rangle=\sum_{x=0}^{2^{n}-1} \frac{1}{\sqrt{2^{n}}}|x\rangle$. In this game, an infinite sequence of operators $\hat{O}_{1} \cdots \hat{O}_{m} \cdots$ will be applied to $|\psi\rangle$. The player decides when to stop the sequence, i.e. he has the freedom to choose $m$ such that $\left|\psi_{f}\right\rangle=\hat{O}_{m} \cdots \hat{O}_{1}|\psi\rangle$. The payoff is then determined by a measurement in the computational basis of $\left|\psi_{f}\right\rangle$. The game is winning if the player possesses a strategy that wins with probability $>1 / 2$, and is losing otherwise. This game incorporates strategic moves, since the set of strategies used by the player to decide the duration of the game are equivalent to the set of natural numbers $\mathbf{N}$.

Game $A$ : Here $\hat{O}_{i}=\hat{A}$ for all $i$, where $\hat{A}(|x\rangle)=(-1)^{\delta_{x \alpha}}|x\rangle$. Geometrically, $\hat{A}$ reflects the vector $|\psi\rangle$ about $|\alpha\rangle$. Since $\hat{A}^{2}=I$, the player's freedom in choosing when to stop the game will always reduce to just one of the following two scenarios: $\left|\psi_{f}\right\rangle=\hat{A}|\psi\rangle$ or $\left|\psi_{f}\right\rangle=|\psi\rangle$. Unfortunately for the player, the payoff $|\langle\alpha|\hat{A}| \psi\rangle|^{2}=|\langle\alpha \mid \psi\rangle|^{2}=\frac{1}{2^{n}}$ which is less than $1 / 2$ for $n \geq 2$. Therefore the player does not possess a winning strategy, hence game A is losing for him.

Game $B$ : Here $\hat{O}_{i}=\hat{B}$ for all $i$, where $\hat{B}:=2|\psi\rangle\langle\psi|-I$. Geometrically, $\hat{B}$ reflects $|\psi\rangle$ about itself. Again, the player has the freedom to decide how many $\hat{B}$ are applied to the input state before measurement. However since $\hat{B}|\psi\rangle=|\psi\rangle$, the player can have no influence in determining the payoff in this game. The game is hence losing for him because the payoff $|\langle\alpha \mid \psi\rangle|^{2}=\frac{1}{2^{n}}$ which is less than $1 / 2$.

Game $A \oplus B$ : The player combines games $\mathrm{A}$ and $\mathrm{B}$ at random. By this we mean $\hat{O}_{i}=\hat{A}$ or $\hat{B}$ with equal probability. Once again, the player has the freedom to decide when to stop the sequence and hence do the measurement. Since $\hat{A}^{2}=\hat{B}^{2}=I$ and $\hat{B}|\psi\rangle=|\psi\rangle$, any given finite sequence $\hat{O}_{i}$ will always produce a final state with the following form: $\left|\psi_{f}\right\rangle=(\hat{B}) \hat{A} \hat{B} \cdots \hat{A} \hat{B} \hat{A}|\psi\rangle$. Now, numerical calculation suggests that for $m=4 k$,

$$
\begin{aligned}
\left|\psi_{f}\right\rangle & =\hat{O}_{m} \cdots \hat{O}_{1}|\psi\rangle \\
& =\overbrace{(\hat{B} \hat{A}) \cdots(\hat{B} \hat{A})}^{k}|\psi\rangle .
\end{aligned}
$$

It can also be seen that $\hat{B} \circ \hat{A}=\hat{G}$ where $\hat{G}$ is Grover's operator 10, 国. Hence a winning strategy for the player is to choose to stop after the 
$4 k$-th operation where $k=\left\lceil\pi \sqrt{2^{n}} / 4\right\rceil$. The winning probability is $>1 / 2$, and hence we see that this combined game is winning for the player.

\section{Conclusion}

We have discussed potentially useful roles for randomness in quantum information processing - in particular, decoherence control and quantum algorithms. The counter-intuitive conclusion is that such randomness/noise might be of direct use in the quantum regime, as opposed to being a guaranteed nuisance. We hope that the present work serves to simulate further research in this fascinating area.

CFL thanks NSERC (Canada), ORS (U.K.) and Clarendon Fund (Oxford) for financial support. NFJ thanks L. Quiroga and F. Rodriguez for discussions. 


\section{References}

[1] M.A. Nielsen and I.L. Chuang, Quantum Computation and Quantum Information (Cambridge University Press, 2000) Chap. 6.

[2] J. Parrondo, G.P. Harmer and D. Abbott, Phys. Rev. Lett. 85, 5226 (2000); G.P. Harmer and D. Abbott, Nature 402, 846 (1999); G.P. Harmer, D. Abbott, P.G. Taylor and J.M.R. Parrondo, Chaos 11, 705 (2001).

[3] E. Lukacs, Characteristic Functions, 2nd ed., revised and enlarged (Griffin, London, 1970), p.18.

[4] R. Bonifacio, S. Olivares, P. Tombesi and D. Vitali, Phys. Rev. A 61, 053802 (2000); R. Bonifacio, Nuovo Cimento Soc. Ital. Fis., B 114, 473 (1999); R. Bonifacio, quant-ph/9901063.

[5] R. Alicki and K. Lendi, Quantum Dynamical Semigroups and Applications (Springer Verlag, Berlin, 1987).

[6] G.J. Milburn, Phys. Rev. A 44, 5401 (1991).

[7] (Closed-loop techniques) P. Tombesi and D. Vitali, Phys. Rev. A 51, 4913 (1995); P. Goetsch, P. Tombesi and D. Vitali, Phys. Rev. A 54, 4519 (1996); D. Vitali, P. Tombesi and G.J. Milburn, Phys. Rev. Lett. 79, 2442 (1997); D. Vitali, P. Tombesi and G.J. Milburn, Phys. Rev. A 57, 4930 (1998).

[8] (Open-loop techniques) L. Viola and S. Lloyd, Phys. Rev. A 58, 2733 (1998); M. Ban, J. Mod. Opt. 45, 2315 (1998); D. Vitali and P. Tombesi, Phys. Rev. A 59, 4178 (1999).

[9] S. Mancini, D. Vitali, P. Tombesi and R. Bonifacio, quant$\mathrm{ph} / 0108011$.

[10] L.K. Grover, Phys. Rev. Lett. 79, 325 (1997). 